\title{
To divide or not to divide, this is the question
}

\author{
Akaki Tsilosani, MD, PhD Tbilisi, Georgia Email:akaki.tsilosani@gmail.com
}

\section{"Clearly the follicular unit is an example where the whole is greater than the sum of its parts."}

\section{Introduction}

In 1996, Seager showed that when single-hair micrografts were generated from breaking down larger follicular units (FUs), their growth was less than when the follicular units were kept intact. ${ }^{2}$ One year later he demonstrated that "skinny" grafts had an $89 \%$ survival rate, whereas "chubby" follicular units had a survival of $113 \%$, presumably due to the fact that hairs in telogen, which were not initially counted, also began to grow. ${ }^{3}$ In 1999 , Beehner compared survival in 60 "skinny" and 60 "chubby" grafts, but left an equal amount of tissue surrounding the dermal papillae. Results showed survival rates of $101 \%$ and $133 \%$, respectively. ${ }^{4}$ It was assumed that the poor growth of several of the singlehair and "skinny" micrografts could be the result of the disruption of the anatomic and physiological links between follicles in naturally grown follicular units, as well as of a higher sensibility of such grafts towards planting trauma and dehydration. We could not have disagreed with the remarks of Bernstein: "Clearly the follicular unit is an example where the whole is greater than the sum of its parts"' and, thus, in the beginning of the twenty-first century, I, as well as many other hair transplant surgeons, firmly believed that follicular units are intact units, and breaking them down was almost a crime. For years we have carried out megasessions in Caucasians solely with follicular units. In Caucasian patients, follicular units contain 1 to 4 follicles (mostly 2-and 3-hair follicles), although occasionally we can see FUs containing more that 4 follicles (Figure 1). According to our experience, Caucasians have a hair/graft ratio of 1.9 and a donor density close to $100 \mathrm{FU} / \mathrm{cm}^{2}$ in the midocciput, ${ }^{5}$ though many authors indicate lower donor densities of $65-85 \mathrm{Fu} / \mathrm{cm}^{2}{ }^{2} .7$ In patients with good scalp laxity and high donor density, harvesting big $50-60 \mathrm{~cm}^{2}$ strips $(32 \mathrm{~cm}$ long and $2.5 \mathrm{~cm}$ wide in the center) can generate around 5,000 FUs.

Working with Asian patients, however, we came across lower donor densities, lower hair/graft ratios, and less scalp laxity. Asian patients have mainly 1-, 2-, and 3-hair follicular units, and 4-hair follicular units were seldom encountered. Imagawa reported that $80 \%$ of Asian grafts consist of 1 or 2 hairs. ${ }^{8}$ And hair/graft ratio in Asians was reported by Pathomvanich as $1.8^{9}$ and by Bernstein as $1.7 .^{10}$ According to our observation, the hair/graft ratio among Asian patients

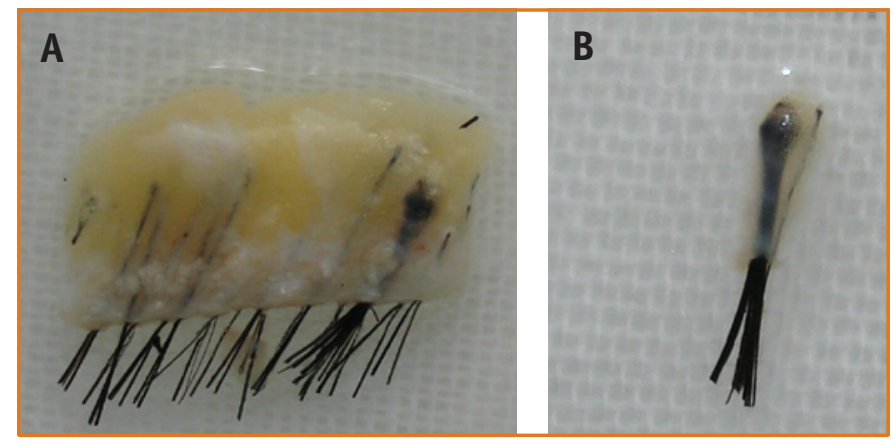

Figure 1. A: 7-hair graft in the sliver; B: 7-hair graft was 1.63 , donor density was $74 \mathrm{FU} / \mathrm{cm}^{2}$, and scalp laxity was 1.5 times less than among Caucasians. ${ }^{5}$ Due to these factors, we were seldom able to generate 3,000 FUs from Asian patients for transplantation and were surprised at hearing about strip transplantation of 4,000 or more grafts in these patients. In our opinion, the only possible way of obtaining grafts at this quantity is through breaking down follicular units into 1- and 2-hair grafts, which increases the quantity of grafts from donor strips obviously containing the same quantity of hair follicles.

Certain hair transplant surgeons confirmed to me in private conversations that they break up follicular units into 1 - and 2-hair grafts and achieve the effect of hair fullness rather than using intact (1-, 2-, 3-, and 4-hair) FUs. At the same time, some studies appear to indicate that it should be safe to divide FUs, if needed..$^{12}$ In 1999, Beehner performed a study comparing intact FUs to grafts with the same number of hair follicles but containing follicles from two adjacent FUs that were subdivided. The grafts containing follicles from subdivided FUs actually had a little better survival, though not significant. ${ }^{4}$

The goal of this study was to see if there is any obvious visible clinical difference in the cosmetic result when transplanting the same quantity of hair follicles, whether transplanting them in natural follicular units or transplanting them as separate hair grafts.

\section{Materials and Methods}

We selected a Caucasian volunteer for the study, a healthy 32-year-old man, with male pattern baldness of Norwood class IVa (Figure 2). We excised a donor strip of $20 \mathrm{~cm}$ long and a maximum $1.5 \mathrm{~cm}$ width in the center with a single-blade knife. The strip was divided into two equal parts. The parts were

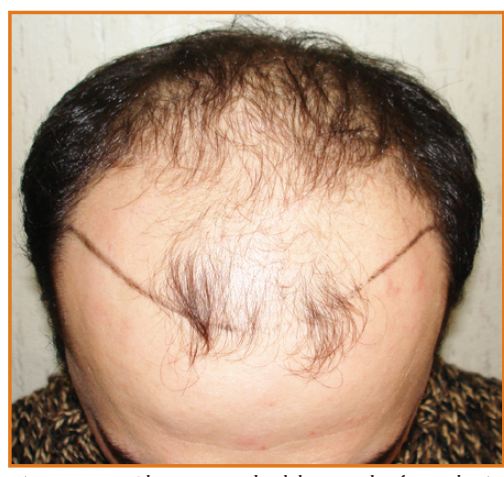

Figure 2. Class IVa baldness before hair transplantation given to two teams with four cutters in each. The first team used MEIJI stereomicroscopes with $20 \times$ zooming capacity and prepared 1,002 intact follicular units (375 1-hair, 462 2-hair, 125 3-hair, and 40 4-hair FUs, totaling 1,834 follicles) with a hair/graft ratio of 1.83 . The second team was instructed to divide all follicular units into 1-hair grafts or, alternatively, into 2-hair grafts if their further division was risky for follicular transection. As a result, 1,404 grafts were generated (1,132 1-hair and 272 2-hair grafts, totaling 1,676 follicles) with a hair/graft ratio of 1.19 . Thus, from one part of the strip we generated 1,002 intact FUs with 1,834 follicles; and from the second part, 1,404 1- to 2-hair grafts with 1,676 follicles. The greater quantity of hair follicles in the first half of the strip was probably the result of the two halves of the strip not being exactly equal in size. 


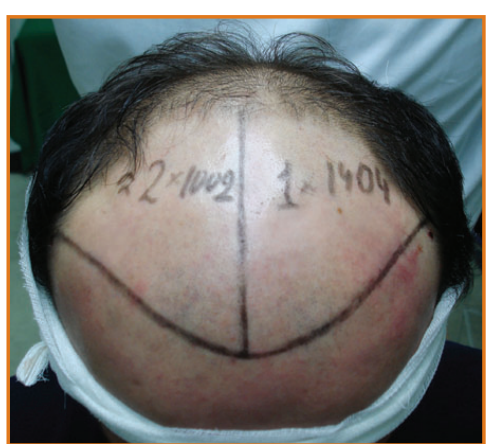

Figure 3. Recipient area divided into 2 equal parts
The recipient area was divided into two equal parts (Figure 3 ). We created 1,002 vertical recipient sites on the right side and 1,404 vertical recipient sites on the left side with 15 degree Sharpoint microblades. These sites were huge for 1 - to 2-hair grafts, but we wanted to have similar sites on both sides for every graft. On the right side of the recipient area, 1,002 intact FUs were implanted (in the first several rows of the hairline 1-hair FUs were implanted, and they were followed by 2-, 3-, and 4-hair FUs). On the left side of the recipient area, 1,404 (mainly 1-hair grafts) were implanted (Figure 4). Recipient sites on both sides were filled simultaneously by two assistants. Recipient area was nearly $80 \mathrm{~cm}^{2}$, so the average density was $25 \mathrm{FUs} / \mathrm{cm}$ on the right side $(1,002$ intact FUs in $40 \mathrm{~cm}^{2}$ ), and 35 grafts $/ \mathrm{cm}^{2}$ on the left side $(1,404$ divided grafts in $40 \mathrm{~cm}^{2}$ ).

\section{Results and Discussion}

The patient was examined 5 months after the operation. Cursory observation did not reveal any obvious difference in hair coverage between the two parts of the recipient area (Fig-

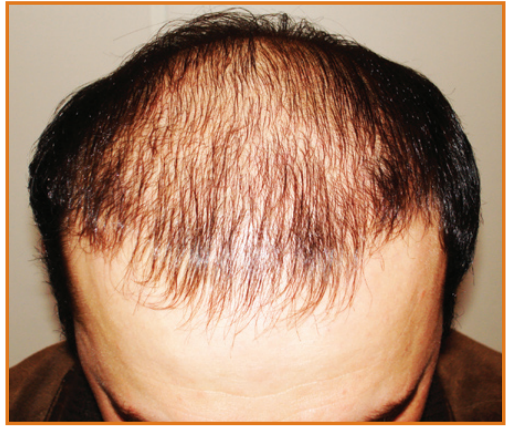

Figure 5. Recipient area after 5 months ure 5), but more careful observation from a close distance seemed to show that the hair grew more naturally and fully on the left side than on the right side, despite 1,676 hair follicles being implanted on the left side, compared to 1,834 on the right. We did not have any reason to consider that the surviv-

ability of the implanted grafts on the left and the right sides of the recipient area were different. Although a greater number of hairs were implanted on the right side, since this hair grew in groups of 2-3 hairs, it gave us the impression of less fullness when observing from a close distance. This difference was not noticeable when viewed from a conversational distance with the hair combed to the side (Figure 6).

We recognize that with the study of only one case we can not draw any firm conclusion. Also, final results are attained in 12 to 18 months. Unfortunately, our patient (case of study) left the country and we lost contact with him so the last photos we have of him are after 5 months. In our experience, at five months, average sessions (2,000-3,000 FUs) yield up to
$70 \%$ of planted hairs.

Therefore, the question remains open: Is it better to divide FUs into 1- or 2-hair follicles to generate more grafts from a certain

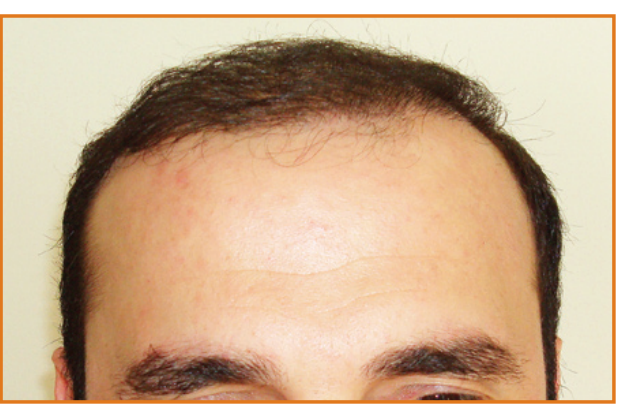

Figure 6. After hair transplantation strip? Through the short history of hair transplant surgery there have been many controversial questions: dissecting microscope versus magnifying loupes, one layer donor closure versus two layer donor closure, skinny versus chubby grafts, staples versus sutures, to trim superior or inferior wound edge during trichophytic closure, sagittal versus coronal slits, etc. Most of these questions are fully answered but many still remain unanswered. Sometimes it is better to combine different methods. It is important that there are no closed topics in our Society and we can raise questions and try to find answers to them through discussion and practical experience over the years.

\section{References}

1. Bernstein R.M, and W.R. Rassman. The logic of follicular unit transplantation. Dermatologic Clinics 1999; 17(2):277-295.

2. Seager, D. Binocular stereoscopic dissecting microscopes: should we use them? Hair Transplant Forum Int'l. 1996; 6(4):2-5.

3. Seager, D. Micrograft size and subsequent survival. Derm Surg. 1997; 23:772-774.

4. Beehner, M. A comparison of hair growth between follicular unit grafts trimmed "skinny" vs "chubby". Derm Surg. 1999; 25(4):339-340.

5. Tsilosani, A., and E. Mshvenieradze. Donor supply of scalp and specificities of hair transplantation in Asians. Georgian Medical News. 2008; 7:12-19.

6. Jimenes, F., and J. Ruifernández. Distribution of human hair in follicular units: a mathematical model for estimating the donor size in follicular unit transplantation. Derm Surg. 1999; 25:294-298.

7. Keene, S.A. Natural hairline density in men: findings of a pilot survey. Hair Transplant Forum Int'l. 2009; 19(2):41-48.

8. Arnold, J. Review of the ISHRS regional workshop: Asian Hair Surgery Workshop hosted by Kenichiro Imagawa. Hair Transplant Forum Int'l. 17(3): 94-95.

9. Pathomvanich, D. Hair Transplantation in Asians. In: Hair Replacement Surgical and Medical. D. Stough and R. Haber, eds. Mosby 2006; 149-156.

10. Bernstein, R.M., and W.R. Rassman. The aesthetics of follicular transplantation. Derm Surg. 1997; 23(9):785-799.

11. Beehner, M. Hair growth in intact follicular units vs non-intact follicular units. 7th Annual Meeting of the International Society of Hair Restoration Surgery. San Francisco. October 1999.

12. Parsley, W.M. Factors influencing graft survival. Hair Transplant Forum Int'l. 2008; 18(4):125-128.

13. Nakatsui, Th., J. Wong, and D. Croot. Survival of density packed follicular unit grafts using the lateral slit technique. Derm Surg. 2008; 8:1016-1022.

14. Rassman, W.R. Is a graft a graft? That is the question! Hair Transplant Forum Int'l. 2006; 1.仓 
To divide or not to divide

$\checkmark$ from page 129

\section{Editorial Comment}

Michael L. Beehner, MD Saratoga Springs, New York

I appreciate Dr. Tsilosani's effort at looking once again at this most perplexing dilemma in hair transplant surgery: Do we place a great many sharply trimmed, very tiny grafts close together or do we instead transplant larger, slightly "chubby" grafts into larger, less densely packed recipient sites? He attempts to look at the survival between intact FU grafts and that of 1- to 2-hair micrografts resulting from dissecting an intact FU, however, my only criticism is that the means used to measure the results are not very objective. We are mostly relying on photographs, with no hair counts or "study boxes" used. I would agree that the one photo with the hair combed forward at 5 months does indeed show roughly an equal visible degree of hair growth on both sides. We know from Dr. David Seager's and my subsequent similar study comparing "chubby" versus "skinny" Fu grafts that additional hairs are realized with the "chubby" ones, but the flip side is that such grafts require larger recipient sites and a lesser density of planting. Larger recipient sites almost certainly are more injurious to the scalp's vasculature. The ideal answer probably lies somewhere between the two extremes. Another point to remember is that leaving the graft a little "chubbier," especially in the upper half just beneath the dermis, helps to preserve the important germinal "bulge" area of the follicle.

Dr. Tsilosani mentioned that in this study he used Sharpoint 15 blades for all the recipient sites, even for the frag- mented 1-hair micrografts. I realize his reasons for doing so, in making the injury equal on the two sides, and find it surprising that he got such apparently good growth with such oversized sites for the 1-hair grafts. Lastly, 5 months is awfully early to make conclusions in any hair-survival or hair-growth study, but I understand the patient moved away and was lost to follow-up.

In his answers to my first review of his article, the author replied: "The question is: Which one will give more visible effect: 3 hairs growing from one point (3-hair FU planted in one recipient site) or 3 hairs growing from three different points (three one-hair grafts obtained by dividing 3-hair graft, planted in three different points)?" My own overwhelming personal experience over the years and also the impressions of Dr. Ron Shapiro in his chapter in the Unger textbook on recipient sites are that hairs coming out of a small tight area (nature's own density) lend a light-blocking quality to the result, which is superior to the cumulative light-blocking ability of the same number of hairs spread out over a wider area. Obviously, the downside of carrying this too far is the "tufty" look that can be imparted, but I think there is an artistic happy medium in which, on selected patients, the final visible density will be greater if double follicular unit (DFU) and triple follicular unit (TFU) grafts are selectively used in inner areas of the frontal and mid-scalp regions.

My hope is that his study will prompt more hair surgeons to conduct further corroborative studies, hopefully with some more objective hair survival modalities used in the study, so that we can arrive at better and more definitive answers to some of these questions.

\section{Editors' Note}

We would like to acknowledge Dr. Beehner for his extensive help in editing this article. We are aware of the fact that subjective evaluation of early results in a single case report does not answer the question posed by the author. We nevertheless publish this paper to acknowledge the timely relevance of this key topic and to encourage further studies with subjective as well as objective evaluations in patients with different hair characteristics.

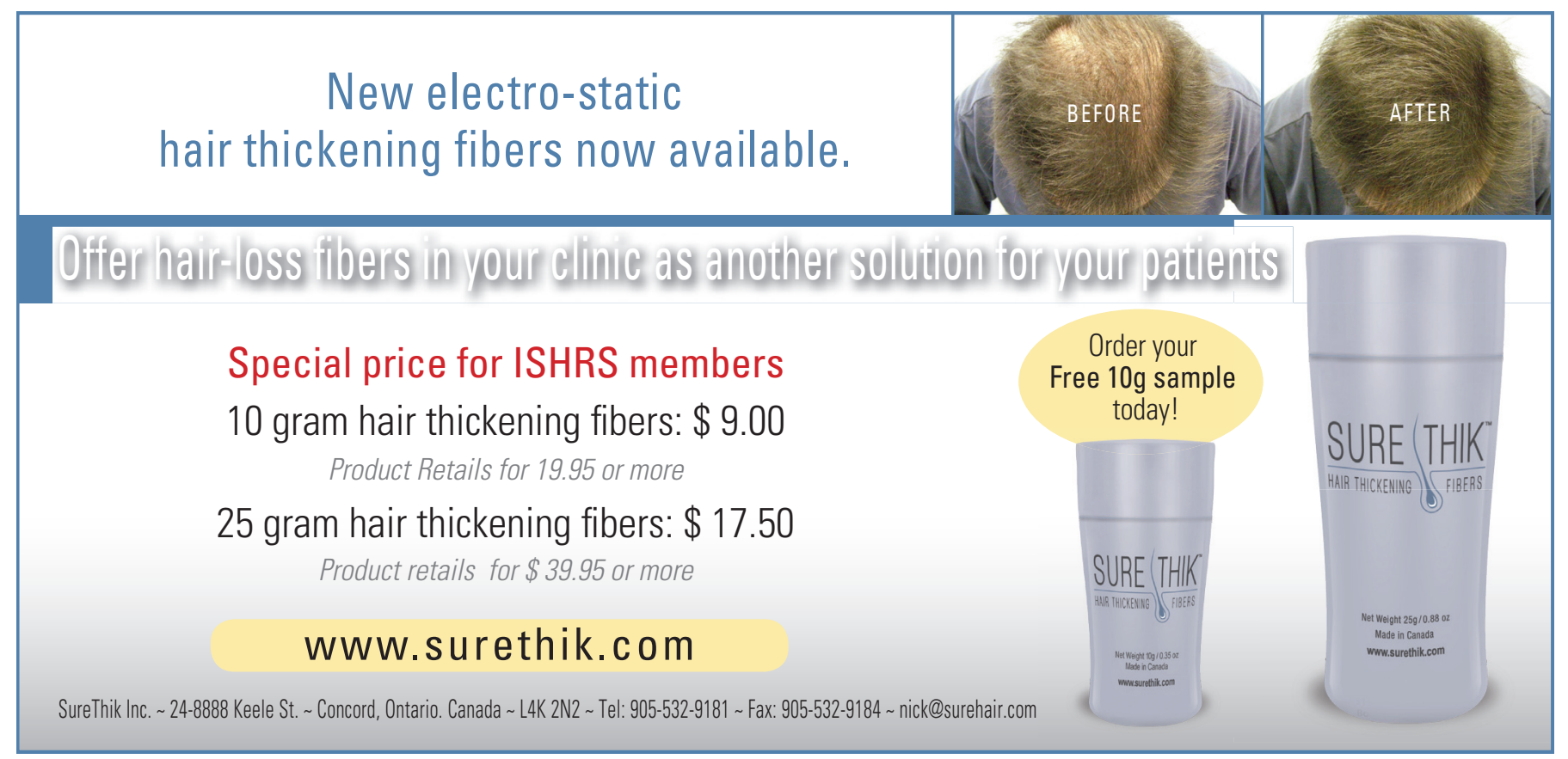

